Gots, J. S., Koн, W. Y. \& HưN, Jun. G. R. (1954). J. gen. Microbiol. 11, 7-16.

\title{
Tryptophan Metabolism and its relation to Phage Resistance in Escherichia coli
}

\author{
By J. S. GOTS, W. Y. KOH and G. R. HUNT, Jun. \\ Department of Microbiology, School of Medicine, University of Pennsylvania, \\ Philadelphia, Pennsylvania, U.S.A.
}

SUMMARY : A series of phage-susceptible tryptophan-requiring mutants of Escherichia coli, strain B, were found to fall into five distinct phenotypes as determined by their alternate growth response to anthranilic acid and indole, and by the substances which they accumulated in the culture fluids. An unidentified substance partially characterized by colorimetric reactions and absorption spectrum accumulated in the culture fluid of two of these types. Tryptophan-requiring $T_{1}$ phage resistant mutants $(B / 1, t r p)$ which were readily obtained by phage selection could not be isolated by the penicillin method. This mutant represents a unique tryptophanless phenotype, not only in terms of its resistance to $T_{1}$ phage, but also on the basis of: (1) accumulation of a new metabolic product; (2) growth stimulation by histidine and tyrosine; (3) suppression of accumulation of certain metabolic products by the other tryptophan auxotrophs; (4) epistatic expression of phenotype when superimposed on the tryptophan auxotrophs by secondary mutation.

In analysing mutants of Escherichia coli, strain B, which are resistant to the $\mathrm{T}$ series of coli phages a consistent and reproducible association is found between resistance to the $T_{1}$ coli phage and a growth requirement for tryptophan (Anderson, 1946). It was felt that an elucidation of the nature of this metabolic lesion might lead to some insight into the mechanism of phage resistance and, by converse analogy, phage susceptibility. In order to determine the nature of the tryptophan requirement of this mutant, we used a method of comparative analysis which utilizes a series of tryptophan auxotrophs derived from the same parent strain by the penicillin selection method (Davis, 1949). The pattern of the tryptophan-deficient phenotypes so obtained involved the same series of genetic blocks in the synthesis of anthranilic acid and its conversion to tryptophan via indole as previously found in mutants of Neurospora (Tatum, Bonner \& Beadle, 1943) and other $E$. coli strains (Davis, 1951). In addition to these previously well-described types of mutants, several new types have been found which are characterized by the excretion of an abnormal, and as yet unidentified, metabolic product.

It was originally hoped that one of the tryptophan auxotrophs would show a resistance to phage $T_{1}$, thus establishing an unequivocal relationship between the resistance and nutritional deficiency. Repeated isolations, however, have revealed no tryptophan auxotrophs which are resistant to $T_{1}$ or any of the other coli phages. The tryptophan-requiring mutant which is resistant to $\mathrm{T}_{1}$ can only be isolated by means of selection for resistance by the action of $T_{1}$ phage. As will be developed here, this organism represents an independent and unique phenotype, not only in terms of its resistance to phage, but also in terms of its tryptophan requirement. 


\section{MATERIAL AND METHODS}

Basal medium. The composition of the defined inorganic salts +glucose medium has been previously described (Gots \& Chu, 1952).

Tryptophan auxotrophs. Mutants of Escherichia coli, strain B, which were unable to grow in the basal medium unless tryptophan was supplied, were obtained by ultraviolet irradiation followed by selection with penicillin (Davis, 1949). These mutants will henceforth be referred to as the 'tryptophan auxotrophs'.

Phage-resistant mutants. Mutants resistant to the action of the $\mathbf{T}$ series of coli phages were obtained by plating approximately $2 \times 10^{8}$ bacteria with sufficient phage to yield confluent lysis. The nomenclature of Demerec \& Fano (1945) will be used to describe these mutants. For example, $B / 1,5$ refers to that mutant of Escherichia coli, strain B, which survived lysis by $\mathbf{T}_{1}$ and was subsequently found to be also resistant to $\mathrm{T}_{5}$.

Accumulations. Cultures were obtained by the growth of the tryptophanrequiring mutants in a suboptimal $(2-5 \mu \mathrm{g} . / \mathrm{ml}$.) concentration of tryptophan for at least $48 \mathrm{hr}$. The bacteria were removed by centrifugation with and without the prior addition of trichloroacetic acid. The detection of accumulated metabolic products in the culture fluid supernatants was accomplished by a variety of methods. Colorimetric analyses were performed with a number of tests for indole and derivatives (Fellers \& Clough, 1925; Gordon \& Weber, 1951), anthranilic acid and derivatives (Kikkawa, 1941), and diazotizable amines (Bratton \& Marshall, 1939). The latter proved the most versatile. It was applied for the quantitative measurement of both anthranilic acid and indole (Eckert, 1943). Separation of the accumulated substances was facilitated by ascending paper chromatography using a $n$-butanol + acetic acid + water (4:1:1) solvent mixture. Ultraviolet spectra were measured with a Beckman quartz spectrophotometer (model DU) and fluorescence was measured with a Klett-Summerson fluorimeter. Syntrophism was detected by auxanographic techniques.

\section{EXPERIMENTAL}

\section{Tryptophan auxotrophs}

A total of thirty-four independent isolations of tryptophan-requiring mutants of Escherichia coli, strain B, could all be classified into three nutritional phenotypes according to the pattern of alternate requirements supplied by anthranilic acid or indole. Type $\operatorname{trp}-1$ (e.g. strain B-106) responded to anthranilic acid or indole, indicating a block in the synthesis of anthranilic acid; $\operatorname{trp}-2$ (e.g. B-37) used indole but not anthranilic acid, indicating a block in the conversion of anthranilic acid to indole; and type $\operatorname{trp}-3$ (e.g. B-82) responded only to tryptophan, indicating an impairment in the conversion of indole to tryptophan. The following substances were unable to replace tryptophan as a growth requirement for any of the organisms: casein acid hydrolysate, $\mathbf{B}$ vitamins, cinnamic acid, indole-3-acetic acid, indole-3-butyric acid, skatole, quinolinic acid, kynurenine,* kynurenic acid, 3-OH-anthranilic acid* and tryptamine.

* We are indebted to Dr D. Bonner for a supply of these compounds. 
In addition to these types of organisms, mutants with a triple requirement for tryptophan, tyrosine and phenylalanine were also found. These represent an early block in the synthesis of a common aromatic precursor (Davis, 1951). Anthranilic acid or indole could replace the tryptophan part of the multiple requirement.

In order to establish the exact nature of the genetic blocks, as well as to determine non-allelism within a group of similar nutritional types, the culture fluids of the auxotrophs were examined for accumulated intermediates. By the methods employed it was shown that some members of $t r p-2$ accumulated anthranilic acid and some members of $\operatorname{trp}-3$ accumulated indole in relatively high concentrations, but no evidence for any accumulated substance could be found with the $\operatorname{trp}-1$ organisms.

Two additional phenotypes were found, $\operatorname{trp}-2 a$ (e.g. strain B-42) and $\operatorname{trp}-3 a$ (e.g. B-81) which differ from the other members of their respective nutritional groups in that they accumulate neither anthranilic acid nor indole, but accumulate an unidentified indole-like substance. This substance appears to be the same in the two types which produce it. It is readily characterized by its tryptophan-like ultraviolet absorption spectrum with a maximum absorption at $280 \mathrm{~m} \mu$. It gives a blue chromogen with the Bratton \& Marshall (1939) test, a positive xanthydrol reaction, and a red colour with the ferric chloride test for indole acetic acid (Gordon \& Weber, 1951). Since it is unable to feed the trp-1 mutants its role as a normal intermediate cannot readily be assessed. Details of chemical analyses, isolation and characterization will be presented in a future publication.

\section{Mutants resistant to $T_{1}$ coli phage}

The types of phage-resistant mutants of Escherichia coli, strain B, have been thoroughly described by Demerec \& Fano (1945), Beale (1948) and Bryson \& Davidson (1951). The majority of the $T_{1}$ resistant mutants were also resistant to $\mathrm{T}_{5}$ coli phage $(B / 1,5)$ and were like the wild type nutritionally. About 10-25\% of the total $T_{1}$ resistant population were susceptible to $T_{5}$ and manifested a requirement for tryptophan $(B / 1, \operatorname{trp})$. An occasional, but rare, $B / 1$ not requiring tryptophan was also found. As has been reported by Bryson \& Davidson (1951), we have also demonstrated a tryptophan-requirement among some of the mutants which are resistant to both $\mathrm{T}_{1}$ and $\mathrm{T}_{5}(B / 1,5, \operatorname{trp})$. These, however, probably represented two-step mutations $(B / 1, \operatorname{trp} / 1 h, 5)$ involving secondary resistance to $T_{1 \mathrm{~h}}$, the host range mutant of $T_{1}$ which is present in $T_{1}$ stocks and mimics $T_{5}$ in its host range activity. This was supported by the fact that a tryptophan-requirement was never found among the $B / 5,1$ mutants isolated by the selective action of $T_{5}$ which is devoid of $T_{1 n}$. Also, when the action of $\mathrm{T}_{\mathrm{Ih}}$ was prevented by citrate (unpublished observation), no tryptophan requirement could be found in any of the $B / 1,5$ mutants isolated by the action of $T_{1}$. Numerous complexities have been revealed in these analyses which, not being important to our present discussion, will be reserved for a future presentation.

All of the $B / 1, \operatorname{trp}$ mutants so far examined fell into nutritional type trp-3 
in that neither indole nor anthranilic acid could replace tryptophan for growth. They differed, however, from the previously described members of this group in that neither indole nor the ' 280 ' substance was accumulated. With the ordinary chemical tests employed, no difference, with respect to accumulation, could be shown between the parent wild type and the $B / 1, \operatorname{trp}$ mutants. However, by spectrophotometric analysis a weak, but distinct, absorption was evident with a peak at $338 \mathrm{~m} \mu$. This is shown in Fig. 1. Attempts to increase

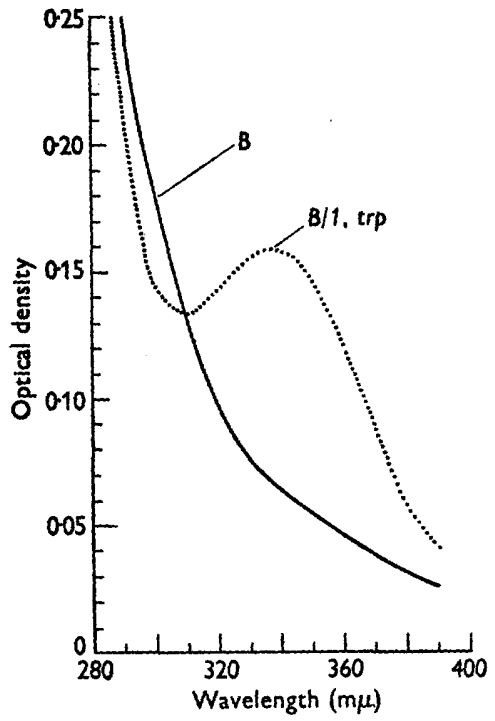

Fig. 1

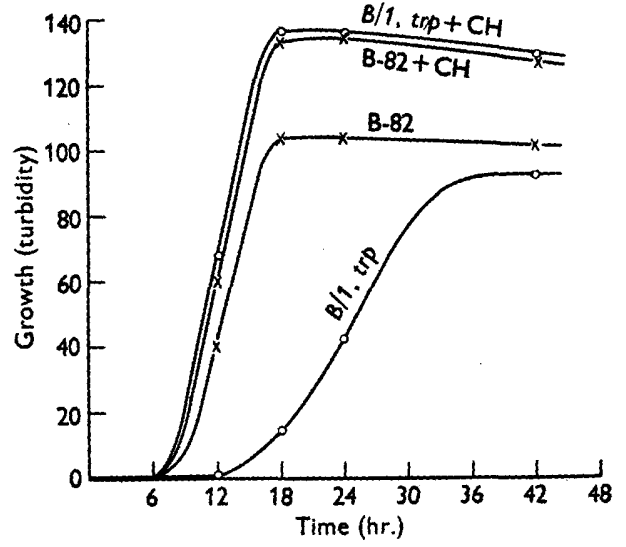

Fig. 2

Fig. 1. Ultraviolet absorption spectra of culture supernatants of Escherichia coli, strains $B$ and $B / 1, t r p$. Supernatants were obtained from $48 \mathrm{hr}$. cultures grown in minimal medium containing $5 \mu \mathrm{g}$. tryptophan $/ \mathrm{ml}$.

Fig. 2. Comparison of growth rates of $B / 1, \operatorname{trp}$ and a $\operatorname{trp}-3$ type (B-82). Media consisted of minimal medium containing $10 \mu \mathrm{g}$. tryptophan $/ \mathrm{ml}$. with and without casein hydrolysate $(\mathrm{CH}), 0.1 \%$.

the concentration of this material by addition of casein hydrolysate, yeast extract or B vitamins were unsuccessful. Its absorption pattern is reminiscent of the absorption of the reduced pyridine nucleus as given, e.g. by reduced diphosphopyridine nucleotide (DPN). That this was not reduced DPN was shown by the use of a bacterial DPN-cytochrome-reductase (Brodie, 1952). The flavoprotein, which rapidly oxidizes reduced DPN with loss of $340 \mathrm{~m} \mu$. absorption, had no effect on the absorption of the $B / 1, \operatorname{trp}$ supernatant.

\section{Growth response to tryptophan}

A striking difference in the type of tryptophan requirement between $B / 1, \operatorname{trp}$ and the other auxotrophic mutants was seen in the nature of the dose-response curves. It can be seen from Fig. 2 that the response of $B / 1, \operatorname{tr} p$ to tryptophan was slower in rate and lower in maximum growth obtained when compared with one of the tryptophan-requiring phage susceptible 
mutants. All of the latter were similar in this respect. This difference shown by the $B / 1, \operatorname{trp}$ mutant was completely nullified in the presence of an amino acid environment such as casein hydrolysate. Similar amino acid effects have been noted by Anderson (1946) and Raff \& Cohen (1950). An analysis of the separate amino acids contained in casein hydrolysate revealed that the entire effect of the latter could be replaced by histidine + tyrosine. As is shown in Fig. 3, histidine acts by increasing the growth rate in a given concentration of tryptophan and the presence of tyrosine permits an elevation of the maximum growth obtained. The mechanism of these stimulations is unknown.

\section{Phage-resistant mutants of the tryptophan auxotrophs}

When the phage-sensitive tryptophan auxotrophs were subjected to the action of $T_{1}$ and $T_{5}$ coli phages a series of phage-resistant mutants were obtained which, in terms of their phage-resistance patterns, were the same as those obtained by the action of the phages on the wild type. These were examined for alterations of the original phenotype as measured by nutritional responses and accumulations. Those mutants which were resistant to both $T_{1}$ and $\mathrm{T}_{5}(\operatorname{trp} / 1,5$ or $\operatorname{trp} / 5,1)$ were indistinguishable from the phenotype of the sensitive parent from which they were derived. However, those which were resistant to $T_{1}$ but not $T_{5}(\operatorname{trp} / 1)$ were no longer phenotypically identical with their respective parents. In all cases, the trp/1 mutant could no longer respond to any of the tryptophan precursors, thus acting as a trp-3 nutritional type even though its original parent may have been a $\operatorname{trp}-1$ or $\operatorname{trp}-2$ type. Furthermore, those organisms which showed accumulations could no longer accumulate these substances when carrying the superimposed mutation to $\mathbf{T}_{1}$ resistance. Thus, a condition of epistasis was evident in that the $\operatorname{trp} / 1$ mutants exhibited a phenotype indistinguishable from that of $B / 1, \operatorname{trp}$ regardless of what the original parent phenotype may have been. A summary of these data is given in Table 1. The $B / 1$ phage-resistant type shown in the table is a $B / 1, \operatorname{trp}$ mutant. Though $B / 1$ which do not require tryptophan have been found, these have not been included in the table since their occurrence is rare and no counterpart has been found among the phage-resistant derivatives of the tryptophan auxotrophs.

The alteration of the nutritional phenotype by the mutation to $\mathbf{T}_{\mathbf{1}}$ resistance is understandable on the basis of the inability of the original $B / 1, \operatorname{trp}$ mutant to respond to the intermediates preceding tryptophan. However, the suppression of the accumulation of the intermediates cannot be explained so readily on the basis of a double genetic block. In reconstruction experiments where an indole accumulator (B-82) and $B / 1, \operatorname{trp}$ were simultaneously inoculated into the minimal medium containing a suboptimal concentration of tryptophan, it was found that the accumulation of indole in the culture fluid was greatly suppressed. This indicated that the $B / 1$, trp organism could prevent the accumulation of indole by strain B-82. More rigorous proof was obtained by the incorporation of sterile culture fluids of $B / 1, \operatorname{trp}$ into medium inoculated with B-82. Fig. 4 shows that such addition to the media markedly depressed the accumulation of indole as compared with controls in which similar 
Table 1. Phenotypes of Escherichia coli involving tryptophan requirement and phage resistance

\begin{tabular}{|c|c|c|c|c|c|c|c|c|c|}
\hline \multirow[b]{2}{*}{ Strain } & \multirow{2}{*}{$\begin{array}{l}\text { Phage- } \\
\text { resistant } \\
\text { phenotype }\end{array}$} & \multicolumn{3}{|c|}{ Phage pattern } & \multicolumn{3}{|c|}{ Growth response } & & \multirow{2}{*}{$\begin{array}{l}\text { Accumulation } \\
\text { of intermediates }\end{array}$} \\
\hline & & $\mathrm{T}_{1}$ & $T_{1 h}$ & $\overrightarrow{T_{5}}$ & o & $\mathbf{A}$ & I & $\mathbf{T}$ & \\
\hline B & $\begin{array}{l}\text { Wild type } \\
B / 1,5 \\
B / 1\end{array}$ & $\begin{array}{l}\mathbf{S} \\
\mathbf{R} \\
\mathbf{R}\end{array}$ & $\begin{array}{l}\mathbf{S} \\
\mathbf{R} \\
\mathbf{S}\end{array}$ & $\begin{array}{l}\mathbf{S} \\
\mathbf{R} \\
\mathbf{S}\end{array}$ & $\begin{array}{l}+ \\
+ \\
-\end{array}$ & + & $\begin{array}{l}+ \\
+ \\
+\end{array}$ & $\begin{array}{l}+ \\
+ \\
+\end{array}$ & $\begin{array}{l}\text { None } \\
\text { None } \\
\text { None }\end{array}$ \\
\hline B-106 & $\begin{array}{l}\operatorname{trp}-1 \\
\operatorname{trp}-1 / 1,5 \\
\operatorname{trp}-1 / 1\end{array}$ & $\begin{array}{l}\mathbf{S} \\
\mathbf{R} \\
\mathbf{R}\end{array}$ & $\begin{array}{l}\mathbf{S} \\
\mathbf{R} \\
\mathbf{S}\end{array}$ & $\begin{array}{l}\mathbf{S} \\
\mathbf{R} \\
\mathbf{S}\end{array}$ & $\begin{array}{l}- \\
-\end{array}$ & + & $\begin{array}{l}+ \\
+ \\
+\end{array}$ & $\begin{array}{l}+ \\
+ \\
+\end{array}$ & $\begin{array}{l}\text { None } \\
\text { None } \\
\text { None }\end{array}$ \\
\hline B-37 & $\begin{array}{l}\operatorname{trp}-2 \\
\operatorname{trp}-2 / 1,5 \\
\operatorname{trp}-2 / 1\end{array}$ & $\begin{array}{l}\mathbf{S} \\
\mathbf{R} \\
\mathbf{R}\end{array}$ & $\begin{array}{l}\mathbf{S} \\
\mathbf{R} \\
\mathbf{S}\end{array}$ & $\begin{array}{l}\mathbf{S} \\
\mathbf{R} \\
\mathbf{S}\end{array}$ & $\begin{array}{l}- \\
-\end{array}$ & - & $\begin{array}{l}+ \\
+ \\
+\end{array}$ & $\begin{array}{l}+ \\
+ \\
+\end{array}$ & $\begin{array}{c}\text { Anthranilic acid } \\
\text { Anthranilic acid } \\
\text { None }\end{array}$ \\
\hline B-42 & $\begin{array}{l}\operatorname{trp}-2 a \\
\operatorname{trp}-2 a / 1,5 \\
\operatorname{trp}-2 a / 1\end{array}$ & $\begin{array}{l}\mathbf{S} \\
\mathbf{R} \\
\mathbf{R}\end{array}$ & $\begin{array}{l}\mathbf{S} \\
\mathbf{R} \\
\mathbf{S}\end{array}$ & $\begin{array}{l}\mathbf{S} \\
\mathbf{R} \\
\mathbf{S}\end{array}$ & $\begin{array}{l}- \\
-\end{array}$ & 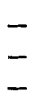 & $\begin{array}{l}+ \\
+ \\
-\end{array}$ & $\begin{array}{l}+ \\
+ \\
+\end{array}$ & $\begin{array}{c}\text { ' } 280 \text { ' substance } \\
\text { ' } 280 \text { ' substance } \\
\text { None }\end{array}$ \\
\hline B-82 & $\begin{array}{l}\operatorname{trp}-3 \\
\operatorname{trp}-3 / 1,5 \\
\operatorname{trp}-3 / 1\end{array}$ & $\begin{array}{l}\mathbf{S} \\
\mathbf{R} \\
\mathbf{R}\end{array}$ & $\begin{array}{l}\mathbf{S} \\
\mathbf{R} \\
\mathbf{S}\end{array}$ & $\begin{array}{l}\mathbf{S} \\
\mathbf{R} \\
\mathbf{S}\end{array}$ & $\begin{array}{l}- \\
- \\
-\end{array}$ & - & I & $\begin{array}{l}+ \\
+ \\
+\end{array}$ & $\begin{array}{l}\text { Indole } \\
\text { Indole } \\
\text { None }\end{array}$ \\
\hline B-81 & $\begin{array}{l}\operatorname{trp}-3 a \\
\operatorname{trp}-3 a / 1,5 \\
\operatorname{trp}-3 a / 1\end{array}$ & $\begin{array}{l}\mathbf{S} \\
\mathbf{R} \\
\mathbf{R}\end{array}$ & $\begin{array}{l}\mathbf{S} \\
\mathbf{R} \\
\mathbf{S}\end{array}$ & $\begin{array}{l}\mathbf{S} \\
\mathbf{R} \\
\mathbf{S}\end{array}$ & $\begin{array}{l}- \\
-\end{array}$ & - & $\underline{-}$ & $\begin{array}{l}+ \\
+ \\
+\end{array}$ & $\begin{array}{c}\text { '280' substance } \\
\text { '280' substance } \\
\text { None }\end{array}$ \\
\hline
\end{tabular}

$S=$ susceptible and $R=$ resistant to phage.

$\mathrm{O}=$ minimal media $; \mathrm{A}=$ minimal plus anthranilic acid $; \mathrm{I}=$ plus indole $; \mathbf{T}=$ plus tryptophan.

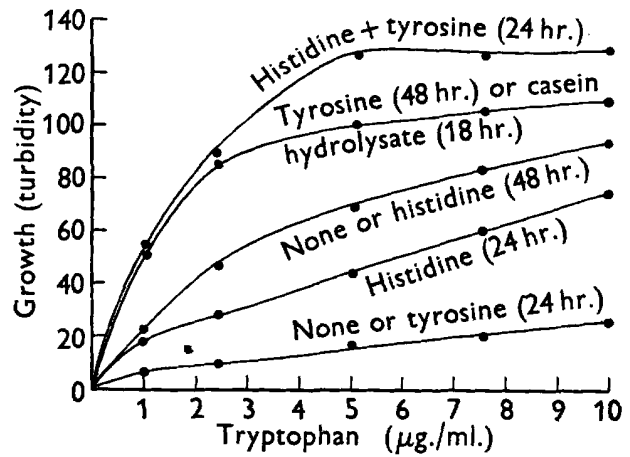

Fig. 3

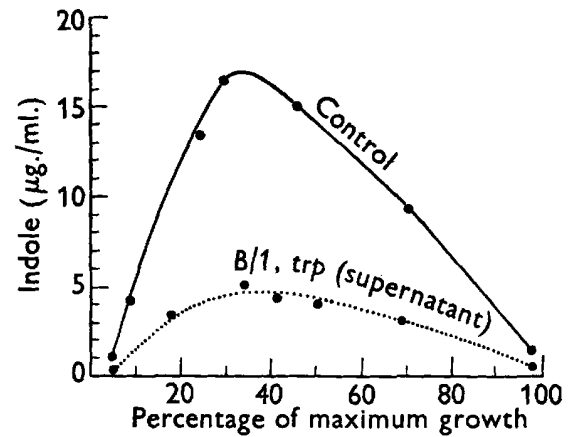

Fig. 4

Fig. 3. Tryptophan dose-response curves of $B / 1, \operatorname{trp}$. Curves are shown for various incubation periods with and without (none) tyrosine $(10 \mu \mathrm{g} . / \mathrm{ml}$. $)$, histidine $(10 \mu \mathrm{g} . / \mathrm{ml}$.) and casein hydrolysate $(0 \cdot 1 \%)$.

Fig. 4. Indole accumulation by strain B-82. Double strength minimal medium was diluted 1:1 with sterile filtrates from $48 \mathrm{hr}$. cultures of $(a)$ wild type grown in minimal medium (control), and (b) $B / 1, t r p$ grown in minimal medium + tryptophan $(5 \mu \mathrm{g} . / \mathrm{ml}$.). These were supplemented with tryptophan in the following concentrations: $0.0,0.1,0.25,0.5$, $1 \cdot 0,2 \cdot 5,5 \cdot 0$ and $10 \cdot 0 \mu \mathrm{g} . / \mathrm{ml}$. After inoculation with strain B-82, percentage of maximal growth was determined from dose-response curves after $48 \mathrm{hr}$. incubation. Indole concentration was measured in supernatants of the $48 \mathrm{hr}$. cultures. 
treatment was made with culture fluids obtained from the wild type. The curves shown were obtained by determining indole concentration in a series of cultures containing increasing amounts of tryptophan. Indole accumulation by this mutant is linear with growth response up to a maximum after which increased growth obtained with increase in tryptophan content results in a suppression of indole accumulation. Since residual tryptophan was present in the supernatants of the $B / 1, \operatorname{trp}$ cultures, its addition would be expected to alter indole accumulation by virtue of the alteration of growth response. It was thus necessary to correlate indole accumulation with percentage of maximum growth as obtained from a tryptophan dose-response curve. With this correction for alteration of growth response it is obvious, as shown in the figure, that indole accumulation by B-82 was markedly suppressed when supernatants from $B / 1, \operatorname{trp}$ were present in the medium. The abscissal displacement of the points shown represent the growth stimulation brought about by the presence of the $B / 1, \operatorname{trp}$ supernatant. Thus, where $1 \mu \mathrm{g}$. of tryptophan per ml. (4th point) allowed $30 \%$ of maximal growth, this was increased to $41 \%$ in the presence of the $B / 1, \operatorname{trp}$ supernatant. Similar experiments with strain B-37 showed that anthranilic acid accumulation is also, but not as markedly, inhibited by $B / 1, \operatorname{tr} p$ filtrates.

\section{DISCUSSION}

The original object of this study was to identify the nature of the tryptophan requirement of the $T_{1}$ resistant mutant by comparison with a series of comparatively well-defined auxotrophs, representing genetic blocks in the sequence of tryptophan synthesis from anthranilic acid. No identity could be established. Instead, the $B / 1, \operatorname{trp}$ mutant was found to be a different tryptophanless phenotype. This was shown by its unique type of accumulation, its growth stimulation by histidine and tyrosine, and its epistatic behaviour when present with other tryptophanless mutations.

It was particularly disturbing to find that though tryptophanless auxotrophs are isolated readily from a population of Escherichia coli they are never found to be phage resistant and always fall into one of five readily classified phenotypes. Repeated attempts to isolate the $B / 1, \operatorname{tr} p$ type by selection for its tryptophan-requiring character have failed. This was true even with populations which were known to be heavily concentrated with respect to $B / 1, \operatorname{tr} p$ as determined by fluctuation analysis and selection for phage resistance.

It is possible that the tryptophan requirement of $B / 1, \operatorname{trp}$ is not due to a deficiency in tryptophan synthesis but may be an expression of a deleterious or growth-limiting reaction which can be neutralized by tryptophan. Examination of the effects of culture filtrates and sonically prepared extracts of $B / 1, \operatorname{trp}$ on the growth of the wild type, the tryptophan auxotrophs and the $B / 1,5$ mutant revealed no inhibitory action. Also, the presence of these fractions did not affect the course of $T_{1}$ production by the wild type. If an inhibitory mechanism is responsible for the tryptophan requirement of $B / 1, \operatorname{trp}$, it is of a nature too subtle to permit experimental detection.

Anderson (1944) has presented a generalized scheme involving dichotomous 
interrelationships between phage resistances and metabolic changes leading to increased growth requirement. From the data presented here we have formulated an extension of the scheme only as a tentative representation to allow for integration of the observed series of phenotypes. Its strength rests on the principles of biochemical genetics which allow for a correlation of phenotypic expressions with postulated genetic breaks.

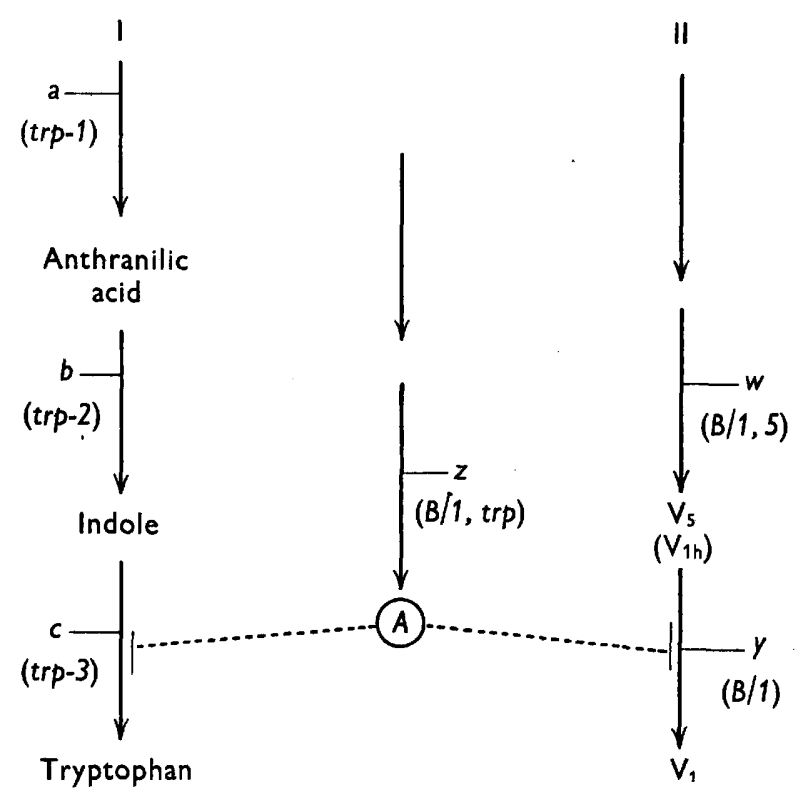

Fig. 5. Tentative scheme depicting mutational sites of observed phenotypes.

Fig. 5 shows two independent pathways, I and II. Pathway I leads to the formation of tryptophan via anthranilic acid and indole. Phage-sensitive tryptophan auxotrophs would result from mutations at sites $a, b$ and $c$ yielding $\operatorname{trp}-1, \operatorname{trp}-2$ and $\operatorname{trp}-3$ types respectively. Location of $\operatorname{trp}-2 a$ and $\operatorname{trp}-3 a$ cannot be made accurately until the nature of the substance which they accumulate is determined.

The other pathway (II) leads to the formation of the factors which must be present to enable the bacterial cell to react with the phages $T_{1}$ and $T_{5}$. Using Anderson's nomenclature (1944), these are referred to as $V_{1}$ and $V_{5}$ respectively. The lack of these factors would render the bacteria resistant to the phages. A mutation at site $w$ would yield the $B / 1,5$ or $B / 5,1$ types, and a mutation at site $y$ would result in the $B / 1$ type. These would all be prototrophic. The $V_{5}$ factor has been depicted as an incomplete $V_{1}$ in order to allow for the fact that no $B / 5$ mutant, to the best of our knowledge, has yet been described. Since the host range of $T_{5}$ and $T_{1 h}$ are identical, $V_{5}$ and $V_{1 h}$ are either the same or very closely related.

This concept is also in accord with the findings of Garen \& Puck (1951) who have examined the first two steps of the invasion of Escherichia coli, strain B, and its $T_{1}$ resistant mutants by the $T_{1}$ phage. The $B / 1,5$ mutant was resistant 
to $T_{1}$ because of the failure of the primary reversible attachment to occur. This step could be controlled by $V_{5}$. The $B / 1$ mutant was able to carry out this reversible attachment $\left(V_{5}\right.$ present), but was unable to permit subsequent irreversible transformation ( $V_{1}$ absent).

In order to explain the $B / 1, \operatorname{trp}$ mutation it is necessary to link the tryptophan pathway with the $V_{1}$ pathway. This can be done by a common factor, ' $\boldsymbol{A}$ ', which places both pathways under unit control. The presence or absence of this factor would be controlled at site $z$. If in the normal cell, $A$ is required both for formation of tryptophan from indole and for completion of $V_{1}$, a 'loss' mutation at $z$ would result in the observed phenotype. However, the same phenotype could be obtained by a 'gain' mutation at $z$ in which case the normally absent $\boldsymbol{A}$ would exercise a suppressive mechanism at the sites indicated. The data available at present cannot definitely distinguish between these two possibilities. In order to explain the stimulatory effects of histidine and tyrosine, as well as the suppression of accumulations, it is also necessary to postulate that a mutation involving $z$ must lead to other metabolic disturbances and imbalances.

The mutant which is resistant to both $T_{1}$ and $T_{5}$ and exhibits a tryptophan requirement cannot be fitted into the postulated scheme as a single mutation. As stated before this probably represents a two-step mutation obtained by secondary selection with $T_{1 h}$. A mutation at $z$ followed by another at $w$ would give this phenotype.

It must be emphasized that the exposition presented above applies only to the tryptophan deficiency found among spontaneously occurring mutants. Bryson \& Davidson (1951), by means of ultraviolet induction, obtained phageresistant mutants of Escherichia coli, strain $B / r$, which exhibited a variety of nutritional requirements. By back mutation analysis involving reversions to prototrophy, many of these have been shown to be multiple phenotypes or double mutants.

This research was aided by a grant from The National Foundation for Infantile Paralysis, Inc.

\section{REFERENCES}

ANDERson, E. H. (1944). Incidence of metabolic changes among virus-resistant mutants of a bacterial strain. Proc. nat. Acad. Sci., Wash. 30, 397.

ANDERson, E. H. (1946). Growth requirements of virus-resistant mutants of Escherichia coli, strain 'B'. Proc. nat. Acad. Sci., Wash. 32, 120.

BEALE, G. H. (1948). A method for the measurement of mutation rate from phage sensitivity to phage resistance in Escherichia coli. J. gen. Microbiol. 2, 131.

Bratton, A. C. \& Marshall, E. K. (1939). A new coupling compound for sulfanilamide determination. J. biol. Chem. 128, 537.

Brodie, A. F. (1952). A bacterial diphosphopyridine nucleotide-linked cytochrome C reductase. J. biol. Chem. 199, 835.

Bryson, V. \& Davidson, H. (1951). Spontaneous and ultraviolet-induced mutations to phage resistance in Escherichia coli. Proc. nat. Acad. Sci., Wash. 37, 784.

Davis, B. D. (1949). The isolation of biochemically deficient mutants of bacteria by means of penicillin. Proc. nat. Acad. Sci., Wash. 35, 1. 
Davis, B. D. (1951). Aromatic synthesis. I. The role of shikimic acid. J. biol. Chem. 191, 315.

Demerec, M. \& Fano, U. (1945). Bacteriophage-resistant mutants in Escherichia coli. Genetics, 30, 119.

Eckert, H. W. (1943). A new micro-colorimetric method for the determination of tryptophane. J. biol. Chem. 148, 205.

Fellers, C. R. \& Clough, R. W. (1925). Indol and skatol determination in bacterial cultures. J. Bact. 10, 105.

Garen, A. \& Puck, T. T. (1951). The first two steps of the invasion of host cells by bacterial viruses. II. J. exp. Med. 94, 177.

Gordon, S. A. \& Weber, R. P. (1951). Colorimetric estimation of indole acetic acid. Plant Physiol. 26, 192.

Gots, J. S. \& ChU, E. C. (1952). Studies on purine metabolism in bacteria. I. The role of $p$-aminobenzoic acid. $J$. Bact. 64, 537.

Kikkawa, H. (1941). Mechanism of pigment formation in Bombyx and Drosophila. Genetics, 26, 587.

RAFF, R. N. \& Conen, S. S. (1950). The effect of virus infection on the utilization of tryptophane in Escherichia coli. J. Bact. 60, 69.

Tatum, E. L., Bonner, D. \& Beadle, G. W. (1943). Anthranilic acid and the biosynthesis of indole and tryptophane by Neurospora. Arch. Biochem. 3, 477.

(Received 3 December 1953) 